\title{
Measuring Individual Regularity in Human Visiting Patterns
}

\author{
Matthew J. Williams, Roger M. Whitaker, and Stuart M. Allen \\ Cardiff School of Computer Science \& Informatics, Cardiff University \\ Queen's Buildings, 5 The Parade, Cardiff CF24 3AA, UK \\ Email: $\{$ m.j.williams, r.m.whitaker, stuart.m.allen\}@cs.cardiff.ac.uk
}

\begin{abstract}
The ability to quantify the level of regularity in an individual's patterns of visiting a particular location provides valuable context in many areas, such as urban planning, reality mining, and opportunistic networks. However, in many cases, visit data is only available as zero-duration events, precluding the application of methods that require continuous, denselysampled data. To address this, our approach in this paper takes inspiration from an established body of research in the neural coding community that deals with the similar problem of finding patterns in event-based data. We adapt a neural synchrony measure to develop a method of quantifying the regularity of an individual's visits to a location, where regularity is defined as the level of similarity in weekly visiting patterns. We apply this method to study regularity in three real-world datasets; specifically, a metropolitan transport system, a university campus, and an online location-sharing service. Among our findings we identify a core group of individuals in each dataset that visited at least one location with near-perfect regularity.
\end{abstract}

\section{INTRODUCTION}

The popularity of devices capable of tracking where individuals have visited (such as GPS-enabled mobile phones) offers both opportunities in providing location-aware commercial services to users and research opportunities in measuring and understanding human mobility behaviour. Furthering our understanding of human visiting patterns is important in diverse areas such as urban planning [1], recommender systems [2], opportunistic networks [3], and limiting the spread of biological and computer viruses [4].

It is difficult to study human mobility without considering its temporal nature. It has been shown that both the ordering of visits and the timing of visits [5] contains information that can be used to build powerful predictors of future behaviour. Furthermore, human behaviour is driven by daily and weekly routine [6], [7]. Although this form of temporal structure is a rich source of information about individual behaviour, there has been little work to examine regularity in individual visiting patterns. Factors such as wealth, profession, lifestyle, and health affect an individual's routine, and therefore his or her mobility patterns. This is likely to give rise to diversity in the population's visiting patterns and regularity. Indeed, diversity has been found to be fundamental to human behaviour, both within the same population and among different populations, even having an evolutionary component [8]. Diversity in visiting regularity may also exist among locations, with some places, such as workplaces, having a natural predisposition for routine.
While collective analysis of behaviour (i.e., focusing on aggregate statistics of large populations of individuals) reveals periodic temporal behaviour [7], [9], it is important to also consider the individual scale (e.g., [10]), focusing on the patterns of individuals from which the collective properties emerge. It is at the individual scale that context-aware computing, user profiling, and personalised recommendations are performed. However, analysis at this scale is more challenging as the data are more sparse and the effects of unpredictable changes in behaviour are more prominent. These effects are smoothed at the collective scale due to the aggregation of many different, but weakly correlated, patterns.

In many real-world systems the visits of users are reduced to instantaneous events, with information about the duration of a stay either unrecorded or ignored. Despite this loss of information, it is still valuable to analyse patterns of visits in these systems. Examples of systems that capture event-based visits include 'checkins' to venues in social networks and location sharing services (for example, Facebook, Foursquare, and Google Latitude), geo-tagged user-contributed content (such as Twitter and Flickr), and electronic ticket payments in metropolitan transport systems (such as the London transport network). With these data there is no clear way to infer the staying time, but nevertheless we are still able to extract interesting patterns from arrival times alone.

In this paper we present a simple and efficient method for measuring regularity in an individual's visits to a location and use it to explore the presence of regularity and routine in real-world data. We define regularity as a visiting pattern that is repeated with a reoccurring time frame (for example, on a week-by-week or day-by-day basis). User visit data such as this is very sparse and consequently challenging to effectively model. This sparsity makes it difficult to apply many established approaches for measuring regularity and periodicity, such as nonlinear time series analysis, harmonic analysis, and recurrence quantification analysis, as these are most effective for time series that are continuous and densely sampled. Although these approaches are unsuitable, in this paper we draw on the large body of relevant work in the neurophysiology community dealing with the problem of finding regularity in event-based data.

The measure we present, named IVI-irregularity (intervisit interval irregularity), is adapted from a synchrony measure used in neural coding [11] (the branch of neurophysiology 
concerned with the coding of information among the neurons in the brain). In the context of neural coding, neurophysiologists deal with ensembles of spike trains, where each train represents the instantaneous electrical pulses (or spikes) of a particular neuron. An ensemble of spike trains is said to exhibit high synchrony if the spikes in the trains occur at similar times. Spikes can be regarded as abstract, zero-duration events; in our case, spikes correspond to visits to a particular location. We use a spike train synchrony measure to quantify the dissimilarity in visits in different weeks; if visits in each week occur at very similar times, then dissimilarity is very low, and thus regularity is high. Throughout this paper we use the terms visit and inter-visit interval (IVI) rather than spike and inter-spike interval, as we are applying these techniques outside the context of neurophysiology.

Using IVI-irregularity we seek to determine the prevalence of regular relationships between individuals and locations and factors that influence the level of regularity. We study these questions using three empirical traces of human mobility, and find that a core subgroup of individuals in each dataset have a number of locations they visit with high regularity. For many applications it is useful to treat regular visits differently to erratic visits. Being aware of these characteristics of human mobility, and being able to effectively measure them, is valuable in many of the aforementioned scenarios.

The rest of this paper is structured as follows. The irregularity measure is formulated in Section II. In Section III the datasets used in the analysis are discussed. The analysis of regularity in these datasets is presented in Section IV. We discuss related work in Section V. Finally, in Section VI we conclude the paper with a summary of the contributions and opportunities for future work.

\section{Measuring Regularity}

We define regularity as repeated routine over time. For example, an individual visiting a location at very similar times each week is considered to have a highly regular pattern for that location. On the other hand, if the individual visits the location at very different times each week it is considered to be very a irregular pattern. Throughout this paper we use weekby-week comparison to determine regularity; however, in the following formulation we generalise this to any window size, denoted by $\omega$.

The measure we introduce quantifies the level of irregularity in an individual's visits to a particular location in a given period of time. Let the chronology of an individual $v$ 's visits to a particular location $l$ be denoted by the ordered sequence of times $C_{v, l}=\left\{t_{i} \mid i=1, \ldots, L\right\}$, where $L$ is the number of $v$ 's visits to $l$. These times are assumed to be offsets from some arbitrary origin, giving values $t_{i} \in\left(0, T_{\max }\right] \forall i=1, \ldots, L$. The chronology is segmented into disjoint windows of duration $\omega$ to build $N$ visit trains. The absolute times of visits are translated to offsets from the start time of their corresponding window; thus, each train has visit times in the interval $(0, \omega]$. We assume $T_{\max }$ and $\omega$ are chosen such that $\omega N=T_{\max }$. We denote the number of visits in the $n$th train with $L_{n}$ and the

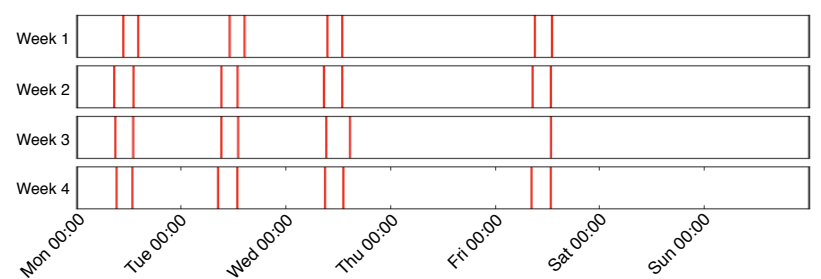

Fig. 1. Example visit trains for a particular user and access point in the DARTMOUTH dataset. Window width $\omega=7$ days.

sequence of visit times with $\left\{u_{i}^{n} \mid i=1, \ldots, L_{n}\right\}$. An example of the visit trains for a chronology in the DARTMOUTH dataset (discussed in Section III) are shown in Figure 1.

Irregularity is quantified by applying the ISI-diversity [12] measure to the ensemble of $N$ visit trains. The measure is computationally efficient, scaling linearly in both the number of visit trains $N$ and number of visits $L$. We begin by defining the inter-visit interval (IVI) as the time between two consecutive visits. The instantaneous inter-visit interval function $I^{n}(u)$ gives the IVI for the $n$th train at time offset $u$; formally, we consider three cases,

$$
\begin{array}{llr}
I^{n}(u)=u_{1}^{n} & \text { if } \quad 0<u \leq u_{1}^{n}, \\
I^{n}(u)=\omega-u_{L_{n}}^{n} & \text { if } & u_{L_{n}}^{n}<u \leq \omega,
\end{array}
$$

and $I^{n}(u)=\min \left(u_{i}^{n} \mid u_{i}^{n} \geq u\right)-\max \left(u_{i}^{n} \mid u_{i}^{n}<u\right)$ if $u_{1}^{n}<$ $u \leq u_{L_{n}}^{n}$.

We define two further instantaneous measures. For time offset $u$, the instantaneous mean $\mu(u)$ is given by

$$
\mu(u)=\frac{1}{N} \sum_{n=1}^{N} I^{n}(u)
$$

and the instantaneous standard deviation $\sigma(u)$ is given by

$$
\sigma(u)=\left(\frac{1}{N-1} \sum_{n=1}^{N}\left(I^{n}(u)-\mu(u)\right)^{2}\right)^{1 / 2} .
$$

The coefficient of variation $c_{v a r}(u)$ provides a measure of dispersion in the IVI values at time offset $u$,

$$
c_{v a r}(u)=\frac{\sigma(u)}{\mu(u)} .
$$

The coefficient of variation is a unitless measure and normalised against the mean, which enables comparison between the dispersion in collections of large IVI values and collections of small IVI values.

By integrating over time offset $u$ we obtain a measure of overall dissimilarity $D\left(C_{v, l}\right)$ in the ensemble of visit trains for chronology $C_{v, l}$; formally,

$$
D\left(C_{v, l}\right)=\frac{1}{\omega} \int_{0}^{\omega} c_{v a r}(u) \mathrm{d} u .
$$

The resulting $D\left(C_{v, l}\right)$ is a non-negative value, with $D\left(C_{v, l}\right)=$ 0 indicating identical trains (or perfect regularity), and higher values indicating more irregularity in the visiting patterns. We refer to $D(\cdot)$ as the IVI-irregularity measure. 


\section{DATASETS}

We use the IVI-irregularity measure to study regularity in visiting patterns in the following datasets.

Foursquare Checkins (FOURSQUARE): Foursquare is a popular location-based mobile social network. Foursquare users voluntarily 'check in' to venues using the Foursquare mobile application. In this way each user compiles a record of his or her visits. We collected a dataset of all checkins in three urban areas in the United Kingdom: Bristol, Cardiff, and Cambridge. These checkins were collected in 2011 [13].

Dartmouth Wireless LAN Access Point Logs (DARTMOUTH): Visits in this dataset are drawn from the use of wireless access points (APs) by staff and students at Dartmouth College campus in the United States [14]. Over 450 APs placed across the $800 \mathrm{~km}^{2}$ of campus provide wireless coverage for most of the area, serving roughly 5,000 undergraduates and 1,200 faculty. The campus includes a variety of facilities, including residences, auditoriums, and social spaces. We use the AP movements trace of April 2003.

London Underground Journeys (UNDERGROUND): The London Underground is a metropolitan rapid-transit rail system serving most of Greater London in the United Kingdom. The Oyster automated fare collection system is used by many passengers, requiring each user to swipe his or her personal Oyster RFID card at the station of entry and station of exit. This provides a record of the passengers' Underground station visits. We obtained an anonymised dataset of all Oyster card journeys over 28 days in March 2010 from Transport for London (TfL), the government body responsible for the service, to use in this paper.

While all three datasets capture visits of individuals to locations, they are drawn from different domains and circumstances, and represent different geographic scales (relevant differences are summarised in Table I). Of the three datasets, FOURSQUARE is unique in that its visits are self-reported by users and so visits may be liable to misreporting and underreporting; nevertheless, it is an interesting dataset as it is at urban scale and covers many venue types. In the case of the DARTMOUTH dataset we carried out additional processing to prepare it for analysis. In particular, we discarded repeated visits by the same user to the same AP separated by a short interval (less than 15 minutes) as these are artefacts of the WLAN AP protocol. In addition, to filter out stationary devices (e.g., wireless-enabled desktop computers), we only included devices that visited at least five different APs.

Each dataset spans a period of four consecutive weeks. We note that the original data contained many individuals that visited certain locations very rarely or exclusively in a few of the four weeks. Chronologies such as these not suitable for studying regularity, as their activity is too rare and too transient. We restrict the datasets to chronologies with at least two visits in each of the four weeks. The resulting datasets are summarised in Table I. This filtering culled $93 \%$ of the original DARTMOUTH and UNDERGROUND person-location pairs, indicating that, although the set of places a person has
TABLE I

SUMMARY OF DATASETS USED IN THE ANALYSIS OF REGULARITY. EACH DATASET CORRESPONDS TO A FOUR-WEEK PERIOD. $M$ DENOTES THE NUMBER OF CHRONOLOGIES AND $\langle L\rangle$ DENOTES THE MEAN NUMBER OF VISITS PER CHRONOLOGY. A CHRONOLOGY $C_{v, l}$ IS ONLY INCLUDED IN A DATASET IF $v$ VISITED $l$ AT LEAST TWICE IN EACH OF THE FOUR WEEKS.

\begin{tabular}{lccc}
\hline \hline Dataset & FoursQuARE & DARTMOUTH & UNDERGROUND \\
\hline \multirow{4}{*}{ Area(s) } & Bristol, & & \\
& Cardiff, and & Dartmouth & London \\
Scale & Cambridge & & \\
Month & Urban & Campus & Metropolitan \\
\hline Location type & June & April & March \\
Visit type & Venue & Access point & Metro station \\
\hline Individuals & Checkin & Association & Card swipe \\
Locations & 293 & 1,681 & $1,167,363$ \\
Visits & 336 & 391 & 270 \\
$M$ & 4,640 & 229,300 & $58,945,475$ \\
$\langle L\rangle$ & 401 & 3,656 & $2,260,354$ \\
\hline \hline
\end{tabular}

visited at least once may be large, many of these places are only visited very occasionally. The number of chronologies for FOURSQUARE reduced to $3 \%$ of the original, leaving a small sample of 401. The remaining chronologies in FOURSQUARE involve $4 \%$ of the users, a small proportion compared to $67 \%$ in DARTMOUTH and $23 \%$ in UNDERGROUND.

\section{Visits AND REGULARITY IN REAL-WORLD MOBILITY TRACES}

We divide our analysis into three areas of interest. We first consider the influence of the time of week on the inter-visit intervals of chronologies (Section IV-A). In Section IV-B we compare the datasets in terms of their irregularity. Finally, we consider how prevalent regular visiting patterns are among the individuals in each dataset (Section IV-C).

\section{A. Inter-visit intervals and the time of week}

As discussed in Section II, our approach focuses on the weekly patterns of inter-visit intervals (IVIs) for an individual's visits to a particular location. The IVIs themselves, along with their level of dispersion at a particular time-ofweek, are an interesting property of human mobility and thus we consider them specifically in Figure 2. The figure shows how IVI dispersion (as quantified by the coefficient of variation $c_{v a r}$ of a chronology at a given time-of-week) varies throughout the week.

The small standard deviations in visit rates indicate that the volume of visits is very similar in each week. This contrasts with the $\left\langle c_{v a r}\right\rangle$ values which have very high standard deviation. This highlights the person-specific nature of an individual's visiting patterns with a location; in other words, the visiting patterns (and therefore IVIs) of two different individuals visiting the same location can be very different.

In the UNDERGROUND dataset we observe that, on average, chronologies' IVIs are most-dispersed between 10:00 and 16:00 on weekdays, and least-dispersed during nighttime. This is because the relative effect of a discrepancy in visit times that are close together is greater than when the visit times are further apart. For example, the morning and afternoon 

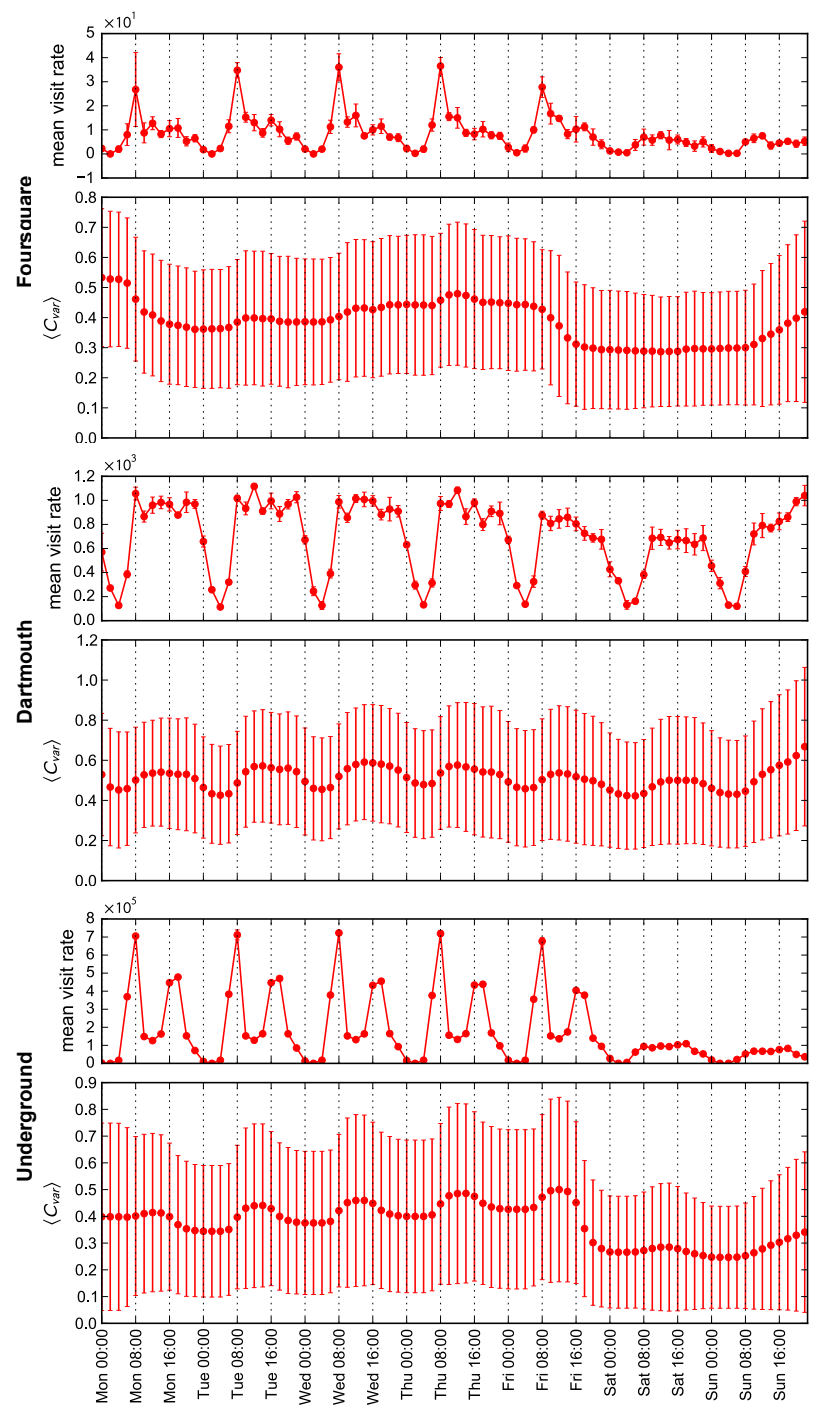

Fig. 2. Time-of-week means of visit rates and coefficients of variation $\left(\left\langle c_{v a r}\right\rangle\right)$ for each dataset. $\left\langle c_{v a r}\right\rangle$ is obtained by averaging over the $c_{v a r}$ values in the corresponding two-hour time slot of all chronologies. A high $\left\langle c_{v a r}\right\rangle$ indicates that the instantaneous IVI values were, on average, more dispersed during that time of week.

commute on the same day are separated by roughly nine hours, whereas the time between the afternoon commute and the following day's morning commute is roughly 15 hours. Therefore, minor discrepancies in the visits to a commuter's stations will have a greater influence on the dispersion of daytime IVIs than nighttime IVIs. The same behaviour is responsible for the dip in IVI dispersion during the weekend. Many chronologies consist of predominantly weekday visits. The weekends for these chronologies will correspond to large IVIs spanning from Friday to Monday, and so the dispersion $\left(c_{v a r}\right)$ will be less during this period.

When comparing DARTMOUTH and UnDERGROUND we note that DARTMOUTH's weekday visit activity is sustained throughout the day and lasts longer into the evening, rarely declining before midnight. This reflects the fact that the

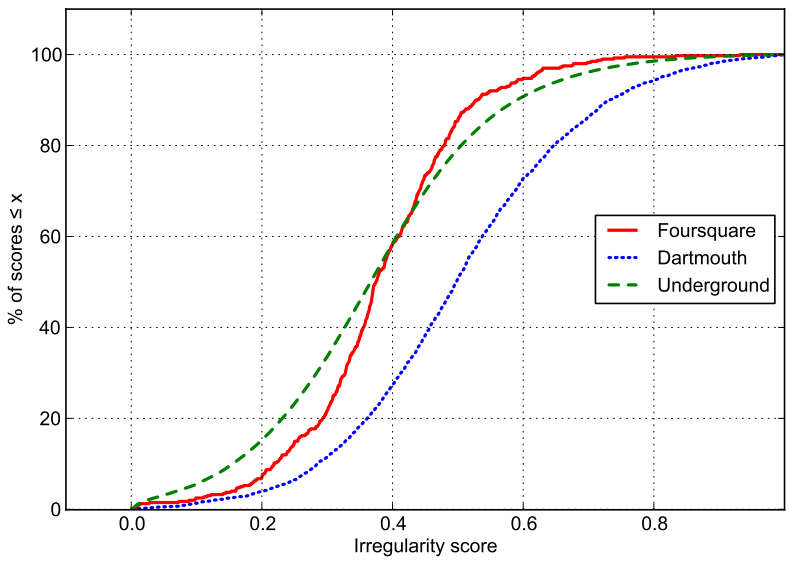

Fig. 3. Cumulative distributions of IVI-irregularity scores (i.e., $D(\cdot)$ values) in each dataset. High $D(\cdot)$ indicates high irregularity. The mean IVIirregularity value $\langle D\rangle$ is $0.381( \pm 0.131)$ for FOURSQUARE, $0.510( \pm 0.185)$ for DARTMOUTH, and $0.373( \pm 0.173)$ for UNDERGROUND.

DARTMOUTH dataset includes many types of visit (including social, residential, and academic), whereas UNDERGROUND is restricted to transportation. This late-evening visit activity is also the reason for the delayed dip in IVI dispersion, which does not decrease until 22:00 (compared to 16:00 in UNDERGROUND). It is also worth noting that the DARTMOUTH decline in visit rate on the weekend is small. This is explained by a large number of students living on-campus, compared to a small proportion of students and staff who either live offcampus or spend the weekend elsewhere.

\section{B. Comparison of regularity between datasets}

Given that the three datasets differ in scale, context, and time of year we would expect differing visiting behaviours in each. Indeed, we have already discussed how the three datasets' time-of-week visit rates exhibit different patterns. The same is also true of the level of regularity present in each dataset, as shown in Figure 3. DARTMOUTH is distinct from the other two datasets, with the weight of its distribution shifted towards higher irregularity. This is reflected in the mean irregularity $\langle D\rangle$ (which we take over the available user-location chronologies), which is higher for DARTMOUTH (0.510) than for FOURSQUARE and UNDERGROUND (0.381 and 0.373 , respectively). This suggests that the patterns of individuals visiting locations on Dartmouth campus tend to be more irregular. This is unlikely to be due to a sudden change in routine, as the duration of the dataset (April 2003) is a continuous period of term-time teaching, uninterrupted by holidays or exams. The small deviations in visit rates (see visit rate plots in Figure 2) also indicate that there was no overall change in visiting patterns between the weeks. An alternative reason for the increased irregularity may be the highly dynamic and spontaneous nature of student behaviour. This contrasts with Underground passengers and Foursquare users, whose student proportion is likely to be much smaller, consisting instead of a large population of individuals following less-flexible routines (for example, commuters). 
The finer-grained scale of the DARTMOUTH dataset may also contribute to the increased irregularity. The Dartmouth APs had an indoor range of around 40 to 100 metres, so most buildings required multiple APs to achieve good WLAN coverage. This means that users moving as little as a few tens of metres can register as having visited a new location. These short-distance movements are likely to be more unpredictable and driven less by routine than larger-distance movements, and thus result in higher irregularity in AP visits.

We also note the similar mean irregularities of FOURQSUARE and UNDERGROUND chronologies, which may be attributed to both datasets being at a city-wide scale and consisting of a broad cross-section of people, as opposed to Dartmouth campus's predominantly student population.

\section{Prevalence of regularity among individuals}

We now study the extent to which an individual has regular relationships with the locations he or she visits. We begin by considering the overall number of locations individuals tend to visit, as shown in Figure 4a. In FOURSQUARE and DARTMOUTH the percentage of individuals decreases with the number of different locations, with DARTMOUTH users typically visiting a wider variety of locations. UNDERGROUND follows a similar pattern, except its peak is at two locations rather than one, which is explained by the nature of Underground journeys. Individuals with only one location are due to the rare instances of a passenger either bypassing the exit turnstile or exiting from the entry station, and the minimumvisits filtering we discussed in Section III.

Using the IVI-irregularity $D\left(C_{v, l}\right)$ of an individual $v$ 's visits to location $l$ we can evaluate whether $v$ 's visits to $l$ are regular or irregular. We set a threshold for irregularity, below which we will regard $v$ 's visits to $l$ as regular. In Figure $4 \mathrm{~b}$ we plot the distribution of individuals and how many of the locations they visited were deemed regular in this way. We set a strict threshold of 0.2 , as we wish to find the chronologies with near-perfect regularity. As shown in Figure 3, a minority of chronologies in each dataset are within this threshold (8.2\% in FourSQUARE, $4.4 \%$ in DARTMOUTH, and $17.4 \%$ in UNDERGROUND).

Figure $4 \mathrm{~b}$ shows how the set of highly regular chronologies is distributed among the individuals. 8\% of Foursquare users and Dartmouth WLAN users had at least one location that they visited with high regularity. The percentage increases in the case of Underground passengers, with $21 \%$ of individuals having at least one regular location, likely due to the moreroutine nature of travel. At stricter thresholds (i.e., thresholds closer to 0), the size of the core group of users with at least one regular venue decreases. The threshold at which the size of this group dropped to $1 \%$ of individuals was 0.009 for FoursQuare, 0.050 for DARTMOUTH, and 0.007 for UNDERGROUND.

We also consider whether there is any relationship between an Underground passenger's most-visited station and his or her most-irregular station. Most-visited stations are likely to be 'home' stations, which we expect to have irregular visiting patterns, since they represent a convolution of many different routines throughout the week. We consider the probability $p(m)$ that, given an individual $v$ who has visited $m$ stations, the individual's most irregular station $l$ (i.e., $l$ such that $D\left(C_{v, l}\right)$ is maximised) is also the station that $v$ visited the most. We find that $p(2)=0.55, p(3)=0.37, p(4)=0.29$, $p(5)=0.28$, and $p(6)=0.28$, indicating that the probability of these stations matching is slightly higher than chance. The deviation from chance becomes greater when individuals have four or more frequently visited stations. This deviation is more significant in DARTMOUTH, which has probabilities $p(2)=0.57, p(3)=0.47$, and $p(4)=0.43$.

\section{RELATED WORK}

Relevant related work includes other approaches to quantifying patterns in human mobility. Information entropy has been used in [15] to quantify the predictability of mobile phone users' patterns of transition between home and work. The work we have presented attempts to go beyond only home and work, considering the many other locations a person visits. An interesting observation in [15] is that university students, especially those in their first year of study, have the highest entropy, and therefore are the least predictable. This agrees with our finding that DARTMOUTH individuals have higher irregularity.

Song et al. [5] have made two key contributions relevant to the work in this paper. First, the authors investigate a different but related concept of regularity, which is defined by them as the probability that an individual is found at his or her mostvisited location. They find that this property is tied to the time-of-week, as we also observed with the mean coefficient of variation (Section IV-A). As previously mentioned, we go beyond the individual's most-visited location and consider their relationships with other places. Second, the authors find that a significant amount of predictive information is encoded in the sequence and ordering of visits. In this paper we have focused on IVIs and their variation by time of week; patterns in the sequences of IVIs is an interesting direction for future work. We also note the datasets in this paper are geographically fine-grained compared to the mobile phone records used in [5], which are on the granularity of cell towers.

There has been research (such as [16]) in the opportunistic networking community that views human behaviour as events in a point process and leverages the corresponding literature; however, we have not found any work in the fields of human mobility or human encounters that utilises the methods of neural coding (in which neuronal spikes are often treated as point processes) as we have in this paper.

\section{CONClusions AND Future Work}

In this paper we introduce a novel method for measuring regularity in an individual's visits to a particular location, adapted from the neural coding concept of synchrony. The method is computationally efficient, does not require binning, and is applicable even for low visit rates. 


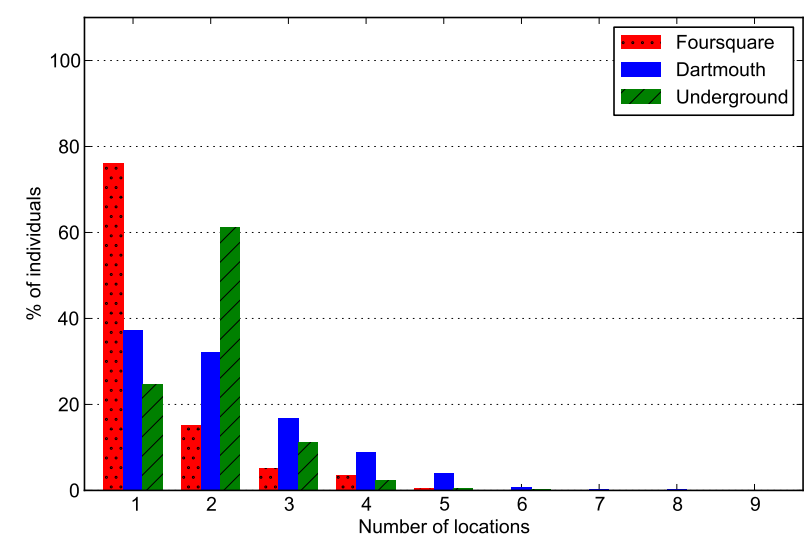

(a) Distributions showing the number of locations for individuals used in our analyses. Individuals exceeding nine locations are not plotted.

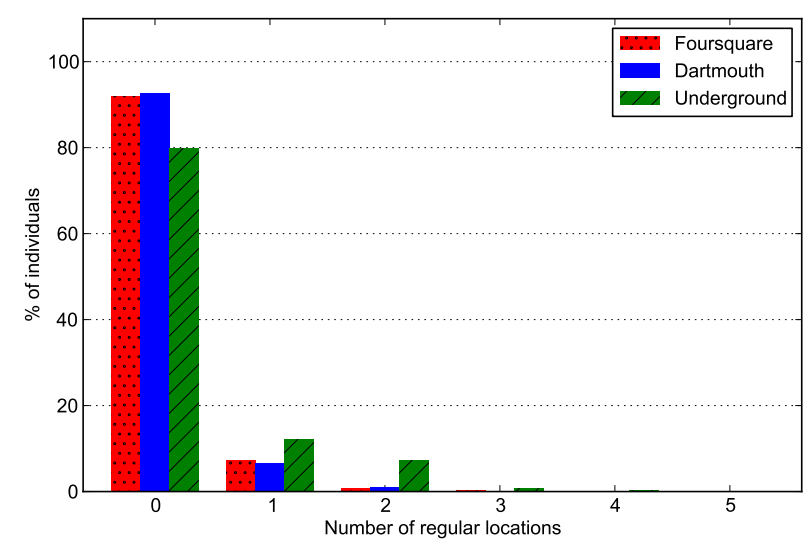

(b) Distributions showing the number of regular locations (i.e., where $\left.D\left(C_{v, l}\right) \leq 0.2\right)$ for individuals in each dataset.

Fig. 4. Number of regular locations per individual compared to the overall number of locations per individual.

Using this method we investigate the visiting patterns of individuals in three diverse datasets; specifically, a metropolitan transport system, a university campus, and an online location-sharing service. We find that campus visits are the most irregular, likely due to the flexible nature of student behaviour, and transport visits are most regular, likely due to the significant commuter population. In all three datasets we find a core group of individuals that visit at least one location with near-perfect regularity. We also note a correlation between an individual's most-visited location (likely to be a associated with their home) and irregularity.

This paper has focused on regularity from the perspective of the individual, but we can use the same approach to consider the location perspective. Future work will investigate how the type of a location (e.g., the Foursquare venue category) influences the regularity of users visiting it, and how this contributes to the overall mean irregularity. We can also consider the prevalence of regularity among locations. This has implications for retailers and shop owners, as it would allow them to distinguish regular visitors from irregular visitors. We intend also to extend this work from person-at-location regularity to person-to-person regularity.

\section{ACKNOWLEDGEMENTS}

We are grateful to Transport for London (TfL), and employees Mark Roberts, Andrew Gaitskell, and Duncan Horne, for providing the London Oyster data. We thank Dafydd Evans for insightful discussions. This research has been funded by RECOGNITION grant 257756, an European Commission FP7 FET project. Data processing was performed in part using the computational facilities of the Cardiff ARCCA division.

\section{REFERENCES}

[1] J. Cranshaw, R. Schwartz, J. Hong, and N. Sadeh, "The livehoods project: Utilizing social media to understand the dynamics of a city," in Proc. 6th Int. AAAI Conf. on Weblogs and Social Media, 2012.
[2] D. Quercia, N. Lathia, F. Calabrese, G. Di Lorenzo, and J. Crowcroft, "Recommending social events from mobile phone location data," in Proc. 10th IEEE Int. Conf. on Data Mining (ICDM), 2010, pp. 971 $-976$.

[3] B. Han, P. Hui, V. Kumar, M. Marathe, J. Shao, and A. Srinivasan, "Mobile data offloading through opportunistic communications and social participation," IEEE Trans. on Mobile Computing, vol. 11, no. 5, pp. $821-834,2012$.

[4] P. Wang, M. C. González, C. A. Hidalgo, and A. Barabási, "Understanding the spreading patterns of mobile phone viruses," Science, vol. 324, no. 5930, pp. 1071-1076, 2009.

[5] C. Song, Z. Qu, N. Blumm, and A. Barabási, "Limits of predictability in human mobility," Science, vol. 327, no. 5968, pp. 1018-1021, 2010.

[6] M. Williams, R. Whitaker, and S. Allen, "Decentralised detection of periodic encounter communities in opportunistic networks," Ad Hoc Networks, 2011, in press.

[7] S. Scellato, M. Musolesi, C. Mascolo, and V. Latora, "On nonstationarity of human contact networks," in Proc. 2nd Workshop on Simplifying Complex Networks for Practitioners, Genoa, Italy, 2010.

[8] G. R. Brown, T. E. Dickins, R. Sear, and K. N. Laland, "Evolutionary accounts of human behavioural diversity," Philosophical Transactions of the Royal Society B: Biological Sciences, vol. 366, no. 1563, pp. 313-324, 2011.

[9] J. Candia, M. C. González, P. Wang, T. Schoenharl, G. Madey, and A. Barabási, "Uncovering individual and collective human dynamics from mobile phone records," Journal of Physics A: Mathematical and Theoretical, vol. 41, no. 22, p. 224015, 2008.

[10] S. Jiang, J. Ferreira, and M. González, "Clustering daily patterns of human activities in the city," Data Mining and Knowledge Discovery, pp. 1-33, 2012.

[11] E. N. Brown, R. E. Kass, and P. P. Mitra, "Multiple neural spike train data analysis: state-of-the-art and future challenges," Nature Neuroscience, vol. 7, no. 5, pp. 456-461, 2004.

[12] T. Kreuz, D. Chicharro, R. G. Andrzejak, J. S. Haas, and H. D. I. Abarbanel, "Measuring multiple spike train synchrony," Journal of Neuroscience Methods, vol. 183, no. 2, pp. 287-299, 2009.

[13] G. B. Colombo, M. J. Chorley, M. J. Williams, S. M. Allen, and R. M. Whitaker, "You are where you eat: Foursquare checkins as indicators of human mobility and behaviour," in Proc. IEEE PERCOM Workshops, 2012.

[14] T. Henderson, D. Kotz, and I. Abyzov, "The changing usage of a mature campus-wide wireless network," Computer Networks, vol. 52, no. 14, pp. 2690-2712, 2008.

[15] N. Eagle and A. Pentland, "Reality mining: sensing complex social systems," Personal and Ubiquitous Computing, vol. 10, no. 4, pp. 255$268,2006$.

[16] A. Chaintreau, P. Hui, J. Crowcroft, C. Diot, R. Gass, and J. Scott, "Impact of human mobility on opportunistic forwarding algorithms," IEEE Trans. on Mobile Computing, vol. 6, no. 6, pp. 606-620, 2007. 